Bull. Korean Math. Soc. 49 (2012), No. 3, pp. 517-528

http://dx.doi.org/10.4134/BKMS.2012.49.3.517

\title{
THE DOMAIN OF ATTRACTION FOR A SEIR EPIDEMIC MODEL BASED ON SUM OF SQUARE OPTIMIZATION
}

\author{
Xiangyong Chen, Chunji Li, Jufang Lü, And Yuanwei Jing \\ ABSTRACT. This paper is estimating the domain of attraction for a class \\ of susceptible-exposed-infectious-recovered (SEIR) epidemic dynamic mo- \\ dels by using sum of squares optimization. First, the stability is analyzed \\ for the equilibriums of SEIR model, and the domain of attraction in the \\ endemic equilibrium is estimated by using sum of squares optimization. \\ Finally, a numerical example is examined.
}

\section{Introduction and preliminaries}

In the past years, the mathematical analysis of epidemic models (Brauer [1], Ma [12]) is often devoted to the problems of the assessment of the asymptotic stability of both the disease free equilibrium and a possible endemic equilibrium. Analysis of steady states of the model and the stability for the epidemic model is of a great importance as it can help our society and direct us to determine and forecast the development trend of infection. The solution results can be used to describe the spread characteristics of infectious diseases, predict the status of the infection and evaluate the efficiency of the control strategies (some contributions are found in $[3,13,23,24]$, where the global asymptotic stability for epidemic models is analyzed in Zhang [24] and Guo [3] etc. Makinde [13] and Zaman [23]) researched the local stability of the equilibrium for epidemic models.

The estimation of domain of attraction (DOA) [10], as an interesting problem in the stability analysis of systems, has been studied by many researchers. In 1967, Hahn [6] proved that the estimation of DOA can be translated into the optimization problem to be solved. As a result, many scholars paid more attention to some optimization problems, in order to estimate DOA, both qualitatively and quantitatively. An algorithm for solving the optimization problems

Received January 4, 2011; Revised March 19, 2011.

2010 Mathematics Subject Classification. 34D20, 92B05, 93D20, 93B40.

Key words and phrases. domain of attraction, SOS optimization, SEIR epidemic model.

The second author was supported by the National Science Foundation of China under Grant 11171301. Also, this work was partially supported by the Fundamental Research Funds for the Central Universities under Grant N100604019 and the National Natural Science Foundation of China under Grant 60774097. 
for estimation of DOA via the approach employing LMI techniques is found in Hachicho and Tibken [5, 4]. Matallana [15] gave a methodology for the estimation of domains of attraction of stable equilibriums based on maximal Lyapunov functions. Important contributions to the estimation of DOA using LMI optimization were given in Chesi [2]. Among some computation methods, those based on Lyapunov functions are dominant in the literatures. These methods compute a Lyapunov function as a local stability certificate and sublevel sets of this Lyapunov function, in which the function decreases along the flow, provide invariant subsets of the DOA.

Sum of squares (SOS) optimization method [19] was introduced to the control community for the purpose of computing the polynomial Lyapunov functions for nonlinear systems. There has been a great interest recently in SOS polynomials and sum of squares optimization, partly due to the fact that these techniques provide convex polynomial time relaxations for many hard problems such as global and constrained optimization, as well as various problems in systems and control. The observation that the SOS decomposition can be computed efficiently using semi-definite programming (Parrilo, [16]) has initiated the development of control application, where Jarvis-Wloszek [8] investigated some controls applications via SOS optimization. Prajna [18] introduced some new developments in SOS method.

Due to the fact that SOS optimization method provides a dynamical iterative algorithm for an optimization Lyapunov function, this dynamic interact mode is more advantage than LMI method. Thereafter many researchers exploited the SOS method to provide lower bounds on the largest estimation of the DOA using convex optimization techniques. Tan [19] presented SOS programs that enlarge a provable region of attraction for polynomial systems. Jarvis-Wloszek [7] estimated the size of the system's region of attraction by finding the largest level set of a Lyapunov function on which the stability theorem's conditions hold using SOS method. Tan and Packard [20] used sum-of-squares programming to obtain the inner bounds of DOAs for dynamical systems with polynomial vector fields. Subsequently, a methodology utilizing information from simulations to generate Lyapunov function candidates satisfying necessary conditions for bilinear constraints is proposed in Topcu and Packard [22], and further innovated in Topcu and Packard [21], where the suitability of the Lyapunov function candidates were assessed solving linear sum-of-squares optimization problems.

Note that infectious disease is a very common phenomenon, in order to analyze the development trend of infectious diseases, the study of the DOA for the epidemic model has also been an active area. $\mathrm{Li}$ [11] presented an optimization approach for finding the DOA of a class SIR models based on the moment theory. Matallana [14] proposed a maximal Lyapunov function approach to estimate the DOA of a class of two-dimensional epidemic models, and the larger estimation of DOA is obtained. Subsequently, Jing and Chen [9] estimated the DOA of an SIRS epidemic model using SOS optimization 
method, the larger domain containing the equilibrium point is obtained by comparing with some other optimization methods.

Thus, in this paper, we introduced the existence of disease-free equilibrium and the endemic equilibrium of a class of SEIR epidemic dynamic models, and analyzed the local stability. By using sum of squares (SOS) optimization, we estimated the DOA. Finally, a numerical example is examined.

Given the following autonomous system

$$
\dot{x}=f(x), \quad x \in \mathbb{R}^{n}
$$

with $f(0)=0$, the domain of attraction (DOA) of $x=0$ is

$$
S_{d}=\left\{x^{0} \in \mathbb{R}^{n} \mid \lim _{t \rightarrow \infty} x\left(t, x^{0}\right)=0\right\},
$$

where $x\left(\cdot, x^{0}\right)$ denotes the solution of (1) corresponding to the initial condition $x(0)=x^{0}$. The following lemmas on finding a domain of attraction using Lyapunov function are a modification of lemmas from [10] and [19].

Lemma 1. Let $V(x)$ be a Lyapunov function defined on a domain $D \subset \mathbb{R}^{n}$ containing the equilibrium point $x=0$ of the system (1), i.e., the following conditions are satisfied

$$
\begin{aligned}
& V(0)=0, \\
& V(x)>0 \text { on } D \backslash(0), \\
& \dot{V}(x)=\nabla V^{*} f<0 \text { on } D \backslash(0) .
\end{aligned}
$$

Then the system (1) is asymptotically stable about $x=0$.

Lemma 2. Let $V(x)$ be a Lyapunov function of the system (1) and let

$$
\begin{aligned}
D_{d} & =\left\{x \in \mathbb{R}^{n} \mid V(x) \leq 1\right\} . \\
\text { If } D_{d} \backslash\{0\} \subseteq\left\{x \in \mathbb{R}^{n} \mid \dot{V}(x)\right. & <0\}, \text { then, } D_{d} \subset S_{d} .
\end{aligned}
$$

Let $R_{n}$ be the set of all polynomials in $n$ variables. We first recall that a polynomial $p(x)=p\left(x_{1}, \ldots, x_{n}\right) \in R_{n}$ is a sum of squares, if there exist polynomials $f_{i}(x)(i=1, \ldots, m)$ such that $p(x)=\sum_{i=1}^{m} f_{i}^{2}(x)$. Let $\Sigma_{n}$ be the set of all SOS polynomials in $n$ variables. The following results are from [19].

Theorem 1. Given polynomials $\left\{f_{1}, \ldots, f_{s}\right\},\left\{g_{1}, \ldots, g_{t}\right\}$, and $\left\{h_{1}, \ldots, h_{u}\right\}$ in $R_{n}$, where $R_{n}$ is the set of all polynomials in $n$ variables, the following assertions are equivalent.

1) The set below is empty:

$$
\left\{\begin{array}{l|l}
x \in \mathbb{R}^{n} & \begin{array}{l}
f_{1}(x) \geq 0, \ldots, f_{s}(x) \geq 0, \\
g_{1}(x) \neq 0, \ldots, g_{t}(x) \neq 0, \\
h_{1}(x)=0, \ldots, h_{u}(x)=0
\end{array}
\end{array}\right\}
$$

2) There exist polynomials $f \in \mathcal{M}\left(f_{1}, \ldots, f_{s}\right), g \in \mathcal{R}\left(g_{1}, \ldots, g_{t}\right), h \in$ $\mathcal{I}\left(h_{1}, \ldots, h_{u}\right)$ such that

$$
f+g^{2}+h=0
$$


where $\mathcal{M}\left(f_{1}, \ldots, f_{s}\right)$ is the multiplicative monoid, $\mathcal{R}\left(g_{1}, \ldots, g_{t}\right)$ is the cone, and $h \in \mathcal{I}\left(h_{1}, \ldots, h_{u}\right)$ is the ideal.

Lemma 3. Given $\left\{p_{i}\right\}_{i=0}^{m} \subset R_{n}$, if there exist $\left\{s_{i}\right\}_{i=1}^{m} \subset \Sigma_{n}$ such that

$$
p_{0}-\sum_{i=1}^{m} s_{i} p_{i} \in \Sigma_{n},
$$

then $\bigcap_{i=1}^{m}\left\{x \in R_{n} \mid p_{i}(x) \geq 0\right\} \subset\left\{x \in R_{n} \mid p_{0}(x) \geq 0\right\}$, that is,

$$
W=\left\{x \in R_{n} \mid p_{1}(x) \geq 0, \ldots, p_{m}(x) \geq 0,-p_{0}(x) \geq 0, p_{0}(x) \neq 0\right\}=\emptyset .
$$

\section{Estimation of DOA of a SEIR model via SOS optimization}

We first review the construction of SEIR model, and then we will show the estimation of DOA model via SOS optimization.

\subsection{SEIR model and the stability}

In recent years, many researchers studied SEIR epidemic model ([24]). In this section, we consider the following SEIR model,

$$
\left\{\begin{array}{l}
\dot{S}=A-\beta S I-\mu S+c I=f, \\
\dot{E}=\beta S I-\mu E-\varepsilon E=g, \\
\dot{I}=\varepsilon E-r I-\mu I-\alpha I-c I=h, \\
\dot{R}=r I-\mu R=q,
\end{array}\right.
$$

where $S(t), E(t), I(t)$ and $R(t)$ are the number of susceptible, exposed, infectious and removed individuals in the population at time $t$, respectively. $\beta$ is the infection rate, $A$ is the recruitment rate of the population, $c$ is the sensible rate without immunity, $\mu$ is the natural mortality rate of the population, $r$ is the recovery rate of infective individuals, $\alpha$ is the death rate due to disease, and $\varepsilon$ is the rate that removed return to the infectious class, and all the parameters are positive constants.

The total population size at time $t$ is $N=S+E+I+R$ satisfying the equation, $\dot{N}=A-\mu N-\alpha I$. When the disease does not exist, the number of the population is tend to the constant $A / \mu$, and when $N>A / \mu, \dot{N}<0$, so all solutions of (3) will remain or tend to the field

$$
D=\left\{(S, E, I, R) \in \mathbb{R}^{4} \mid 0<S+E+I+R \leq \frac{A}{\mu}, S \geq 0, I \geq 0, R \geq 0\right\} .
$$

We denote the basic reproduction number

$$
R_{0}=\frac{A \beta \varepsilon}{\mu(\mu+\varepsilon)(\mu+\alpha+r+c)},
$$

which determines whether the disease dies out or remains. Firstly, the equilibrium points can be solved easily as follows, where the disease-free equilibrium point is $P_{1}(A / \mu, 0,0,0)$, and the endemic equilibrium point is $P_{2}\left(p_{1}, p_{2}, p_{3}, p_{4}\right)$, 
where $p_{1}=\frac{A}{\mu R_{0}}, p_{2}=\frac{A\left(R_{0}-1\right)(\mu+\alpha+r+c)}{R_{0}[\mu(\mu+\alpha+r+c)+\varepsilon(\mu+\alpha+r)]}, p_{3}=\frac{\varepsilon p_{2}}{\mu+\alpha+r+c}$, and $p_{4}=$ $\frac{r \varepsilon p_{2}}{\mu(\mu+\alpha+r+c)}$.

We analyze the asymptotic stability of the disease-free equilibrium and the endemic equilibrium of the SEIR epidemic model.

Theorem 2. If $R_{0}<1$, then $P_{1}$ is the unique equilibrium point of (3), and it is globally asymptotically stable; if $R_{0}>1$, then $P_{1}$ is unstable.

Proof. Let

$M=\left(\begin{array}{cccc}\frac{\partial f}{\partial S} & \frac{\partial f}{\partial E} & \frac{\partial f}{\partial I} & \frac{\partial f}{\partial R} \\ \frac{\partial g}{\partial S} & \frac{\partial g}{\partial E} & \frac{\partial g}{\partial I} & \frac{\partial g}{\partial R} \\ \frac{\partial h}{\partial S} & \frac{\partial h}{\partial E} & \frac{\partial h}{\partial I} & \frac{\partial h}{\partial R} \\ \frac{\partial q}{\partial S} & \frac{\partial q}{\partial E} & \frac{\partial q}{\partial I} & \frac{\partial q}{\partial R}\end{array}\right)=\left(\begin{array}{cccc}-\mu-I \beta & 0 & c-S \beta & 0 \\ I \beta & -\mu-\varepsilon & S \beta & 0 \\ 0 & \varepsilon & -\mu-r-\alpha-c & 0 \\ 0 & 0 & r & -\mu\end{array}\right)$.

For $P_{1}$, we have

$$
\left.M\right|_{P_{1}}=M_{1}=\left(\begin{array}{cccc}
-\mu & 0 & c-\frac{A}{\mu} \beta & 0 \\
0 & -\mu-\varepsilon & \frac{A}{\mu} \beta & 0 \\
0 & \varepsilon & -\mu-r-\alpha-c & 0 \\
0 & 0 & r & -\mu
\end{array}\right) .
$$

The characteristic equation is,

$$
(\lambda+\mu)^{2}\left((\lambda+\mu+\varepsilon)(\lambda+\mu+r+\alpha+c)-\frac{\beta A \varepsilon}{\mu}\right)=0 .
$$

Thus the eigenvalues of $M_{1}$ are $\lambda_{1}=\lambda_{2}=-\mu$, and the others $\lambda_{3}, \lambda_{4}$ satisfy

$$
\lambda^{2}+(2 \mu+\varepsilon+r+\alpha+c) \lambda+(\mu+\varepsilon)(\mu+r+\alpha+c)-\frac{\beta A \varepsilon}{\mu}=0 .
$$

So, when $R_{0}<1$, we know that $(\mu+\varepsilon)(\mu+r+\alpha+c)>\frac{\beta A \varepsilon}{\mu}$, then by RouthHurwitz criterion and $P_{1}$ is unique, we know that $P_{1}$ is globally asymptotically stable. When $R_{0}>1$, we know that $\lambda_{3} \lambda_{4}<0$, and thus $P_{1}$ is unstable.

Theorem 3. If $R_{0}>1$, then $P_{2}$ is one of the equilibrium points of $(3)$, which is locally asymptotically stable.

Proof. For $P_{2}$, we have

$$
\left.M\right|_{P_{2}}=M_{2}=\left(\begin{array}{cccc}
-\mu-p_{3} \beta & 0 & c-p_{1} \beta & 0 \\
p_{3} \beta & -\mu-\varepsilon & p_{1} \beta & 0 \\
0 & \varepsilon & -\mu-r-\alpha-c & 0 \\
0 & 0 & r & -\mu
\end{array}\right) .
$$

The characteristic equation is $(\lambda+\mu)\left(\lambda^{3}+a_{1} \lambda^{2}+a_{2} \lambda+a_{3}\right)=0$, where

$$
\begin{aligned}
& a_{1}=\beta p_{3}+3 \mu+\varepsilon+r+\alpha+c>0, \\
& a_{2}=\mu\left(\beta p_{3}+2 \mu+\varepsilon+r+\alpha+c\right)+\beta p_{3}(\mu+r+\alpha+c+\varepsilon)>0, \\
& a_{3}=\mu \beta p_{3}(\mu+r+\alpha+c+\varepsilon)+\beta \varepsilon p_{3}(\alpha+r)>0 .
\end{aligned}
$$


Since

$$
\begin{aligned}
\Delta_{1}= & a_{1}>0, \\
\Delta_{2}= & a_{1} a_{2}-a_{3}=d_{0} \beta^{2} I^{2}+d_{1} \beta I+d_{2}>0, \\
d_{0}= & c+r+\alpha+2 \mu+\epsilon, \\
d_{1}= & r^{2}+\alpha^{2}+c^{2}+7 \mu^{2}+\epsilon^{2}+(2 c+5 \mu+\epsilon) r \\
& +(2 c+2 r+5 \mu+\epsilon) \alpha+(5 \mu+2 \epsilon) c+5 \mu \epsilon, \\
d_{2}= & \mu(c+r+\alpha+2 \mu+\epsilon)(c+r+\alpha+3 \mu+\epsilon), \\
\Delta_{3}= & a_{3} \Delta_{2}>0,
\end{aligned}
$$

by Routh-Hurwitz criterion, we know that $P_{2}$ is locally asymptotically stable when $R_{0}>1$.

\subsection{Estimation of DOA of SEIR model}

Since there is no the variable $R$ in the three equations of system (3), and some people concern about the popular features of the disease, so we can only analyze the behavior of $S, E$ and $I$ by using the following systems, in which the behavior of $R$ can be obtained by studying $I$,

$$
\left\{\begin{array}{l}
\dot{S}=A-\beta S I-\mu S+c I=f, \\
\dot{E}=\beta S I-\mu E-\varepsilon E=g, \\
\dot{I}=\varepsilon E-r I-\mu I-\alpha I-c I=h .
\end{array}\right.
$$

Then, the positive invariant set about the system (4) is

$$
D=\left\{(S, E, I) \in \mathbb{R}^{3} \mid 0<S+E+I \leq \frac{A}{\mu}, S \geq 0, I \geq 0\right\}
$$

and the equilibrium points of system (4) are $P_{10}(A / \mu, 0,0)$, and $P_{20}\left(p_{1}, p_{2}, p_{3}\right)$.

By Theorem 2 and Theorem 3 , we can know that $P_{20}$ is locally asymptotically stable, when $R_{0}>1$. Let $x=S-p_{1}, y=E-p_{2}$, and $z=I-p_{3}$. Then $P_{20}$ is translated into $P_{20}^{\prime}(0,0,0)$ and the system (4) can be written as follows

$$
\left\{\begin{array}{l}
\dot{x}=-\left(\beta p_{3}+\mu\right) x-\left(\beta p_{1}-c\right) z-\beta x z, \\
\dot{y}=\beta p_{3} x+\beta p_{1} z-(\mu+\varepsilon) y+\beta x z, \\
\dot{z}=\varepsilon y-(\mu+r+\alpha+c) z .
\end{array}\right.
$$

Then, the estimation of DOA in $P_{20}$ can be translated into the estimation of DOA of (5) in $P_{20}^{\prime}$.

To fit the assumptions of Lemma 1 into an SOS programming framework, we restrict $V(x)$ with $V(0)=0$ and describe $D_{0}$ with a semi-algebraic set

$$
D_{0}=\left\{(x, y, z) \in \mathbb{R}^{3} \mid p(x, y, z) \leq \rho\right\}
$$

with $p(x, y, z) \in \Sigma_{3}$, and $\rho \geq 0$ to insure that $D_{0}$ is connected and contains the origin. Now the requirements of Lemma 1 for asymptotic stability become

$$
D_{0} \backslash\{0\} \subseteq\left\{(x, y, z) \in \mathbb{R}^{3} \mid V(x, y, z)>0\right\},
$$


and

$$
D_{0} \backslash\{0\} \subseteq\left\{(x, y, z) \in \mathbb{R}^{3} \mid \dot{V}(x, y, z)<0\right\} .
$$

In order to find the largest estimation of the region of attraction, we will fix $p$ and maximize $\rho$ subject to the above Lyapunov conditions. We pose the following optimization to search for $V$ using the set emptiness form of the set containment constraints above.

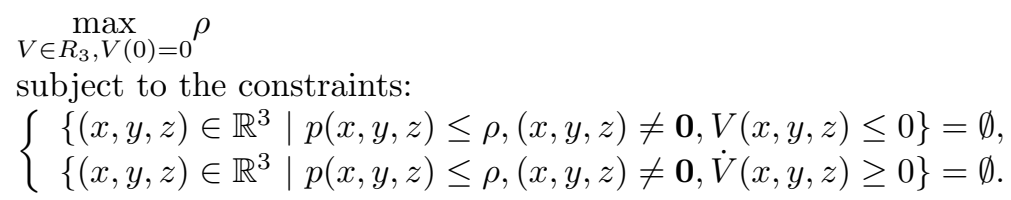

By Lemma 2, if we define

$$
D_{d}=\left\{(x, y, z) \in \mathbb{R}^{3} \mid V(x, y, z) \leq 1\right\}
$$

for some as of yet unknown candidate Lyapunov function, if $D_{d} \backslash\{0\} \subseteq\{(x, y, z)$ $\left.\in \mathbb{R}^{3} \mid \dot{V}(x, y, z)<0\right\}$, then $D_{0}$ must be contained in $D_{d}$. The problem of finding the best estimation of the region of attraction in this framework can be written with set emptiness constraints as

$$
\begin{aligned}
& \max _{V \in R_{3}, V(0)=0} \rho \\
& \text { subject to the constraints: } \\
& \left\{\begin{array}{l}
\left\{(x, y, z) \in \mathbb{R}^{3} \mid(x, y, z) \neq \mathbf{0}, V(x, y, z) \leq 0\right\}=\emptyset, \\
\left\{(x, y, z) \in \mathbb{R}^{3} \mid p(x, y, z) \leq \rho, V(x, y, z)>1\right\}=\emptyset, \\
\left\{(x, y, z) \in \mathbb{R}^{3} \mid V(x, y, z) \leq 1,(x, y, z) \neq \mathbf{0}, \dot{V}(x, y, z) \geq 0\right\}=\emptyset .
\end{array}\right.
\end{aligned}
$$

These conditions are not yet semi-algebraic as they contain the non-polynomial constraint $(x, y, z) \neq \mathbf{0}$. We can get around this problem by using $l_{1}, l_{2} \in \Sigma_{3}$, and replacing $(x, y, z) \neq \mathbf{0}$ with $l_{i}(x, y, z) \neq 0, i=1,2$ to get

$$
\begin{aligned}
& \max _{V \in R_{3}, V(0)=0} \rho \\
& \text { subject to the constraints: } \\
& \left\{\begin{array}{l}
\left\{(x, y, z) \in \mathbb{R}^{3} \mid V(x, y, z) \leq 0, l_{1}(x, y, z) \neq 0\right\}=\emptyset, \\
\left\{(x, y, z) \in \mathbb{R}^{3} \mid p(x, y, z) \leq \rho, V(x, y, z) \geq 1, V(x, y, z) \neq 1\right\}=\emptyset, \\
\left\{(x, y, z) \in \mathbb{R}^{3} \mid V(x, y, z) \leq 1, \dot{V}(x, y, z) \geq 0, l_{2}(x, y, z) \neq 0\right\}=\emptyset .
\end{array}\right.
\end{aligned}
$$

By Theorem 1, the equivalence of the above constraints are the existence of $s_{1}, \ldots, s_{10} \in \Sigma_{3}$ and $k_{1}, k_{2}, k_{3} \in \mathbb{Z}_{+}$satisfying

$$
\begin{aligned}
& \max _{V \in R_{3}, V(0)=0, k_{1}, k_{2}, k_{3} \in \mathbb{Z}_{+}, s_{i} \in \Sigma_{3}, i=1, \ldots, 10} \rho \\
& \text { subject to the constraints: } \\
& \left\{\begin{array}{l}
s_{1}-V s_{2}+l_{1}^{2 k_{1}}=0 \\
s_{3}+(\rho-p) s_{4}+(V-1) s_{5}+(\rho-p)(V-1) s_{6}+(V-1)^{2 k_{2}}=0, \\
s_{7}+(1-V) s_{8}+\dot{V} s_{9}+(1-V) \dot{V} s_{10}+l_{2}^{2 k_{3}}=0 .
\end{array}\right.
\end{aligned}
$$


To make the problem in a form amenable to SOSTOOLs (Prajna, [18] and [17]), we pick $k_{1}=k_{2}=k_{3}=1, s_{2}=l_{1}$ and $s_{1}=l_{1} \hat{s}_{1}$ and factor out $l_{1}$; set $s_{3}=s_{4}=0$ and factor out a $(V-1)$ term; set $s_{10}=0$ and factor out $l_{2}$. After these simplifications, we have

$$
\begin{aligned}
& \max _{V \in R_{3}, V(0)=0, s_{6}, s_{8}, s_{9} \in \Sigma_{3}} \rho \\
& \text { subject to the constraints: } \\
& \left\{\begin{array}{l}
V-l_{1} \in \Sigma_{3} \\
-\left((\rho-p) s_{6}+(V-1)\right) \in \Sigma_{3} \\
-\left((1-V) s_{8}+\dot{V} s_{9}+l_{2}\right) \in \Sigma_{3} .
\end{array}\right.
\end{aligned}
$$

Note that in the above constraints, $V$ is underbounded by $l_{1}$, a positive definite function, so $V$ is positive definite. Furthermore, the set $D_{d}:=\{(x, y, z) \in$ $\left.\mathbb{R}^{3} \mid V(x, y, z) \leq 1\right\}$ is bounded. The following method is used to estimate the DOA.

Algorithm 1. An iterative search to expand the region $D_{0}$ and thus the region $\left.D_{d}:=\left\{(x, y, z) \in \mathbb{R}^{3} \mid V(x, y, z) \leq 1\right)\right\}$ in Lemma 2 starting from a positive definite candidate Lyapunov function.

Step 1. Set $V=V^{(i-1)}$ and solve the linesearch on $\beta$ where $s_{6} \in \Sigma_{3, d_{s_{6}}}$, $s_{8} \in \Sigma_{3, d_{s_{8}}}, s_{9} \in \Sigma_{3, d_{s_{9}}}$,

$$
\begin{aligned}
& \max _{s_{6}, s_{8}, s_{9} \in \Sigma_{3}} \rho \\
& \text { subject to the constraints: } \\
& \left\{\begin{array}{l}
-\left((\rho-p) s_{6}+(V-1)\right) \in \Sigma_{3} \\
-\left((1-V) s_{8}+\dot{V} s_{9}+l_{2}\right) \in \Sigma_{3} .
\end{array}\right.
\end{aligned}
$$

Set $s_{8}^{(i)}=s_{8}, s_{9}^{(i)}=s_{9}$. Continue to Step 2 .

Step 2. Set $s_{8}^{(i)}=s_{8}, s_{9}^{(i)}=s_{9}$, and solve the linesearch on $\beta$ where $V \in R_{3, d_{V}}$ with $V(0)=0$ and $s_{6} \in \Sigma_{3, d_{s_{6}}}$.

$$
\begin{aligned}
& \max _{V \in R_{3}, s_{6} \in \Sigma_{3}} \rho \\
& \text { subject to the constraints: } \\
& \left\{\begin{array}{l}
V-l_{1} \in \Sigma_{3}, \\
-\left((\rho-p) s_{6}+(V-1)\right) \in \Sigma_{3}, \\
-\left((1-V) s_{8}+\dot{V} s_{9}+l_{2}\right) \in \Sigma_{3} .
\end{array}\right.
\end{aligned}
$$

Set $\beta^{(i)}=\beta, V^{(i)}=V$. If $\beta^{(i)}-\beta^{(i-1)}$ is less than a specified tolerance go to Step 3, else increment $i$ and go to Step 1 .

Step 3. The set $\left.D_{d}:=\left\{(x, y, z) \in \mathbb{R}^{3} \mid V(x, y, z) \leq 1\right)\right\}$ contains $D_{0}$ and is the largest estimate of the fixed point's region of attraction.

Remark 1. The degree of $V, d_{V}$, is even. Since the $s$ 's and the l's are SOS, they are all even degree. And for the first SOS constraint, it holds that $d_{V}=d_{l_{1}}$, 
for the second SOS constraint, it holds that $\operatorname{deg}\left(p s_{1}\right) \geq d_{V}$, and for the third SOS constraint, it holds that $\operatorname{deg}\left(V s_{2}\right) \geq \operatorname{deg}\left(\dot{V} s_{3}\right), \operatorname{deg}\left(V s_{2}\right) \geq d_{l_{2}}$.

\section{Numerical example}

In this section, a numerical example of SEIR epidemic model to show the feasibility of SOS optimization method proposed in this paper will be analyzed. We take $A=4, \alpha=r=\mu=\varepsilon=c=\frac{1}{4}, \beta=\frac{1}{2}$. Then $R_{0}=4>1$. The model can be written as

$$
\left\{\begin{array}{l}
\dot{S}=4-\frac{1}{2} S I-\frac{1}{4} S+\frac{1}{4} I, \\
\dot{E}=\frac{1}{2} S I-\frac{1}{2} E \\
\dot{I}=\frac{1}{4} E-I .
\end{array}\right.
$$

And the coordinate of the point $P_{2}$ is $\left(4, \frac{48}{7}, \frac{12}{7}\right)$. Let $S=x+4, E=y+\frac{48}{7}, I=$ $z+\frac{12}{7}$. Then we obtain

$$
\left\{\begin{array}{l}
\dot{x}=-\frac{31}{28} x-\frac{7}{4} z-\frac{1}{2} x z \\
\dot{y}=\frac{6}{7} x+2 z-\frac{1}{2} y+\frac{1}{2} x z, \\
\dot{z}=\frac{1}{4} y-z .
\end{array}\right.
$$

According to Algorithm 1, setting the algorithm stopping tolerance to $\beta^{(i)}-$ $\beta^{(i-1)}=0.01$ and the degrees of $V, l_{1}, l_{2}, s_{6}, s_{8}, s_{9}$ as follows:

$$
d_{V}=2, d_{s_{6}}=d_{s_{8}}=2, d_{s_{9}}=0, d_{l_{1}}=2, d_{l_{2}}=4 .
$$

Then we can obtain a Lyapunov function $V(x, y, z)$ as follows:

$\left.V(x, y, z)\right|_{(7)}=0.0553 x^{2}+0.0827 x y-0.023 x z+0.05 y^{2}+0.0293 y z+0.1023 z^{2}$ and $\rho_{\max }=9.4200$. Thus a subset of the domain of attraction of (7) is

$$
D_{d}=\left\{(x, y, z) \in \mathbb{R}^{3}|V(x, y, z)|_{(7)} \leq 1, x \geq-4, y \geq-\frac{48}{7}, z \geq-\frac{12}{7}\right\} .
$$

As the subset of DOA obtained is an irregular sphere, these simulation results are depicted in Figure 1, where Fig.(1a) shows the subset $D_{d}$ of DOA for the system (7) in the equilibrium point $(0,0,0)$. In order to give a better visualization of the results, we also give the level curves in planes of $x O y$ (Fig.1b), $x O z$ (Fig.1c) and $y O z$ (Fig.1d). It is observed from these figures that the estimated region $D_{d}$ approximates the actual $S_{d}$ near its stability boundary, and it can be ensured that any initial state within $D_{d}$ will end up in an endemic situation.

\section{Conclusions}

In this paper, we discuss the stability of an SEIR epidemic model in the disease-free equilibrium and the endemic equilibrium. Furthermore, we use sum of squares (SOS) optimization method to estimate the DOA in the endemic equilibrium point. The viability of all these ideas is confirmed by the successful application to the model. In such situations, the dominating steady state (infection-free or endemic) will depend on the initial distribution of individuals (susceptible, exposed, infected and removed) in the population and 


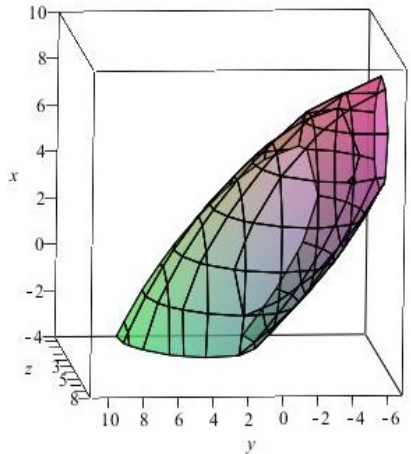

(1a)

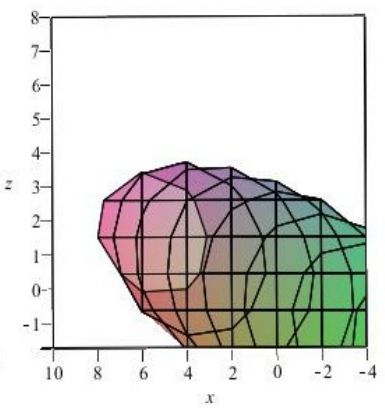

(1c)

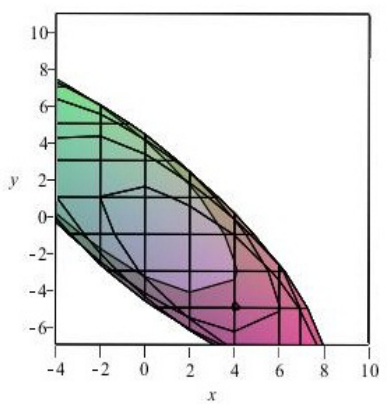

(1b)

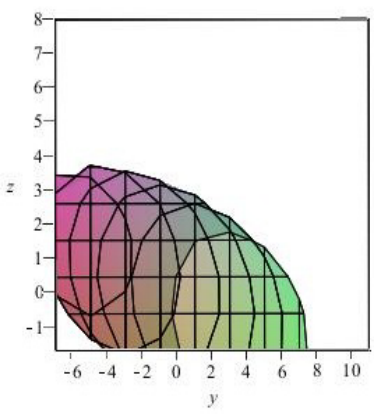

(1d)

Figure 1 . The subset $D_{d}$ of DOA for SEIR system (7)

estimations of the corresponding DOA would be valuable to analyze the spread outcome of the disease. Finally, a numerical example is given to demonstrate feasibility and validity of the proposed technique as well as reveal that this algorithm is better in applications to the epidemic model.

\section{References}

[1] F. Brauer and C. Castillo-Chavez, Mathematical Models in Population Biology and Epidemiology, Springer-Verlag, New York, 2001.

[2] G. Chesi, Estimating the domain of attraction for non-polynomial systems via LMI optimization, Automatica. 45 (2009), 1536-1541.

[3] H. Guo, M. Li, and Z. Shuai, Global stability of the endemic equilibrium of multigroup SIR epidemic models, Canad. Appl. Math. Quart. 14 (2006), no. 3, 259-284. 
[4] O. Hachicho, A novel LMI-based optimization algorithm for the guaranteed estimation of the domain of attraction using rational Lyapunov functions, J. Franklin Inst. 344 (2007), no. 5, 535-552.

[5] O. Hachicho and B. Tibken, Estimating domains of attraction of a class of nonlinear dynamical systems with LMI methods based on the theory of moments, In Proc. CDC. Las Vegas, Nevada. 2002, 3150-3155.

[6] W. Hahn, Stability of Motion, Springer Verlag, Berlin, 1967.

[7] Z. Jarvis-Wloszek, Lyapunov based analysis and controller synthesis for polynomial systems using sum-of squares optimization, $\mathrm{Ph}$. D. Thesis. dissertation. Pasadena, CA. 2003.

[8] Z. Jarvis-Wloszek, R. Feeley, W. Tan, K. Sun, and A. Packard, Some controls applications of sum of squares programming, In Proc. CDC. Hawaii, USA (2003), 4676-4681.

[9] Y. W. Jing, X. Y. Chen, C. J. Li, et al., Domain of attraction estimation for SIRS epidemic models via Sum-of-Square Optimization, August 28 - September 2, in Proc. IFAC WC. Milano, Italy. 2011.

[10] H. Khalil, Nonlinear Systems, 3rd ed. Prentice Hall, 2002.

[11] C. Li, C. Ryoo, N. Li, and L. Cao, Estimating the domain of attraction via moment matrices, Bull. Korean Math. Soc. 46 (2009), no. 6, 1237-1248.

[12] Z. Ma, Y. Zhou, and W. Wang, Mathematical Modeling and Research of Epidemic Dynamical Systems, Beijing, Science Press, 2004.

[13] O. Makinde, A domain decomposition approach to a SIR epidemic model with constant vaccination strategy, Appl. Math. Comput. 184 (2007), no. 2, 842-848.

[14] L. G. Matallana, A. M. Blanco, and J. A. Bandoni, Estimation of domains of attraction in epidemiological models with constant removal rates of infected individuals, 16th argentine bioengineering congress and the 5 th conference of clinical engineering, Journal of Physics: Conference Series. 90 (2007), Art. no. 012052.

[15] Estimation of domains of attraction: a global optimization approach, Math. Comput. Modelling 52 (2010), no. 3-4, 574-585.

[16] P. A. Parrilo, Structured semidefinite programs and semialgebraic geometry methods in robustness and optimization, Ph. D. dissertation. Pasadena, CA. 2000.

[17] S. Prajna, A. Papachristodoulou, P. Seiler, and P. A. Parrilo, SOSTOOLS: Sum of squares optimization toolbox for MATLAB, Available from http://www.cds.caltech. edu/sostools and http://www.aut.ee.ethz.ch/parrilo/sostools, 2002.

[18] S. Prajna, A. Papachristodoulou, P. Seiler, et al. New developments in sum of squares optimization and SOSTOOLS, In Proc. ACC. Boston, USA (2004), 5608-5611.

[19] W. Tan, Nonlinear control analysis and synthesis using sum-of-squares programming, Ph. D. dissertation. UC, Berkeley. 2003.

[20] W. Tan and A. Packard, Stability region analysis using polynomial and composite polynomial Lyapunov functions and sum-of-squares programming, IEEE Trans. Automat. Control 53 (2008), no. 2, 565-571.

[21] U. Topcu, A. Packard, and P. Seiler. Local stability analysis using simulations and sumof-squares programming, Automatica J. IFAC 44 (2008), no. 10, 2669-2675.

[22] U. Topcu, A. Packard, P. Seiler, and T. Wheeler. Stability region analysis using simulations and sum-of-squares programming, In Proc. ACC. New York, USA. (2007), 60096014.

[23] G. Zaman, Y. Kang, and I. Jung, Stability analysis and optimal vaccination of an SIR epidemic model, Biosystems. 93 (2008), 240-249.

[24] J. Zhang, J. Li, and Z. Ma, Global dynamics of an SEIR epidemic model with immigration of different compartments, Acta Math. Sci. Ser. B Engl. Ed. 26 (2006), no. 3, $551-567$. 
Xiangyong Chen

School of Information Science And EngineEring

NortheAstern UNIVERSITY

Shenyang, 110-819, P. R. China

E-mail address: xiangyongchen@yahoo.cn

Chunji Li

Institute of System Science

College of Sciences

NoRThEASTERN UNIVERSITY

ShenyAng, 110-819, P. R. ChinA

E-mail address: chunjili2000@yahoo.com.cn

JUFANG LÜ

College of Sciences

Northeastern University

Shenyang, 110-819, P. R. China

E-mail address: jufang41@163.com

YUANWEI JING

School of Information Science And EngineERING

NoRThEASTERN UNIVERSITY

Shenyang, 110-819, P. R. China

E-mail address: ywjjing@mail.neu.edu.cn 